A method for determining effective elastic constants of a composite unidirectionally reinforced with two types of transtropic hollow fibers is developed. Determining these characteristics is an integral step in the design of composite structures. The approach is based on analytical formulas for determining the elastic characteristics of a two-component composite with a transtropic matrix and hollow fiber. Hexagonal fiber lay-up with periodic reinforcement structure is considered. Double homogenization is used. The composite is conventionally divided into hexagonal regions of two types. The first is a hollow fiber of one material and the surrounding matrix. Similarly, the second one - with a hollow fiber of another material. In the first homogenization, elastic constants of the transtropic material of each of the two regions are determined. In the repeated homogenization, the region of the first type is taken as a "conditional" fiber, the region of the second type is taken as a "conditional" matrix. Effective elastic constants for a composite reinforced with two types of isotropic hollow fibers are calculated. The proposed method gives a good convergence of the results with calculations by known formulas. The maximum relative calculation error for the longitudinal elastic characteristics compared to knozen formulas does not exceed $0.05 \%$. The dependences of some effective elastic constants on the volume content of hollow fibers of various types are constructed. Using this approach, three-component composites can be modeled varying the materials of the matrix, hollow fibers and their volume content. This allows predicting the strength of such composites under certain deformations at the design stage

Keywords: three-component unidirectional composite, transtropic hollow fibers, effective elastic constants, homogenization
UDC 539.3

DOI: $10.15587 / 1729-4061.2021 .242398$

\title{
HOMOGENIZATION OF A UNIDIRECTIONAL COMPOSITE REINFORCED WITH TWO TYPES OF TRANSTROPIC HOLLOW FIBERS
}

\author{
Anastasi a S toliarova
}

Corresponding author

$\mathrm{PhD}$

Department of Software Engineering*

E-mail: st_av991@ukr.net

Andri y $\mathbf{P}$ oz huy e v

$\mathrm{PhD}$, Associate Professor

Department of Fundamental and Applied Mathematics*

Oksana Spytsia

$\mathrm{PhD}$, Associate Professor

Department of General Mathematics*

Alla Bohuslavska

$\mathrm{PhD}$, Associate Professor

Department of Information Technology in Tourism

Zaporizhzhia Polytechnic National University

Zhukovskoho str., 64, Zaporizhzhia, Ukraine, 69063

*Zaporizhzhia National University

Zhukovskoho str., 66, Zaporizhzhia, Ukraine, 69600

Received date 22.08.2021

Accepted date 08.10.2021

Published date 28.10.2021
How to Cite: Stoliarova, A., Pozhuyev, A., Spytsia, O., Bohuslavska, A. (2021). Homogenization of a unidirectional composite reinforced with two types of transtropic hollow fibers. Eastern-European Journal of Enterprise Technologies, 5 (7 (113)), 52-58. doi: https://doi.org/10.15587/1729-4061.2021.242398

\section{Introduction}

Composites are widely used in mechanical engineering, aircraft, rocket and other industries due to their properties and performance characteristics. Composites are applied extensively in the creation of transport, tourist infrastructure, etc. One way to reduce the material consumption of composites is to use hollow fibers. Studies confirm the advantage of such fibers as reinforcing elements over continuous ones under certain types of loads.

Composites are the basis of many materials and structures, which is an important factor in economic development.

Composite design involves many parameters that can be varied to create materials with predefined properties. Manufacturing all possible composite options and conducting field tests is a very expensive procedure. Therefore, before making test samples, their number should be significantly limited, discarding unacceptable ones. This can be done using modern computer-aided design systems that model composites in real operation. One of the modeling steps is composite homogenization, that is, presenting inhomogeneous material as homogeneous with certain "averaged" characteristics, called effective.
The presence of several fiber types also complicates the procedure for obtaining effective elastic constants. So, analytical relations for such composites are found using a large number of hypotheses that reduce the mathematical complexity of the problem. Homogenization greatly simplifies calculations, since taking into account the characteristics and arrangement of each fiber in a mathematical model increases the cost of machine and time resources by orders of magnitude, and sometimes makes such calculations impossible.

The more properties of components are taken into account in the homogenization process, the more adequate the relations will be for the effective characteristics of the composite. Thus, for some fiber types, transverse and longitudinal elastic characteristics may differ by one to two orders of magnitude. So, considering the transtropic properties of the components is relevant and of considerable practical interest.

\section{Literature review and problem statement}

The determination of effective elastic constants of hollow fiber composites is addressed in the papers based on exper- 
imental and theoretical research methods. In particular, two-phase fiber composites are considered. Recent works include [1-7].

Thus, in [1], local stresses in a hollow fiber composite are investigated by the numerical method. It was found that the change in hollow fiber wall thickness affects more the transverse characteristics of the composite than the longitudinal ones. The study [2] is devoted to comparing the results of experimental tests of hollow and continuous fiber-reinforced composites. In particular, tensile, compression, elongation and impact strength tests were performed. A comparison of continuous and hollow fibers as reinforcing elements in the impact strength test of composites is given in [3]. The study is carried out by the finite element method. The same method was used in [4] to estimate the effective longitudinal and transverse Young's moduli of hollow and continuous fiber composites. The results of experimental studies on impact tests of unidirectional composites reinforced with hollow, continuous and mixed polyester fibers are also presented in [5]. The paper [6] provides a review of the creation and use of hollow glass and carbon fiber composites. In particular, such composites have been shown to exhibit high mechanical characteristics.

The studies confirm the advantage of hollow fiber reinforcement over continuous one. However, it should be noted that [1-6] used experimental or numerical methods, which does not allow for analysis of the dependence of effective elastic constants on the parameters of the composite components. Each study was conducted for specific composites. In this case, a large number of new calculations or field tests are required for each new set of component parameters. This is a certain disadvantage compared to analytical methods, since using analytical formulas makes it possible to automate the calculation of effective elastic constants, and perform calculations by varying the input composite parameters.

In [7], general relations are obtained for calculating effective elastic characteristics of transtropic piezoelectric hollow fiber composites. The representative elementary volume method is used. However, these relations do not have a closed analytical form, which greatly complicates their use in calculating effective characteristics.

For two-component composites unidirectionally reinforced with hollow fibers, there are known relations for determining basic effective elastic characteristics. These formulas were obtained by various scientists using the sequential regularization method and the mixture method. However, both groups of relations take into account only the isotropic properties of the composite components. It is clear that adding at least one more fiber type will significantly complicate the mathematical model of the composite behavior even under simple deformations. Consider also the fact that increasingly reinforcing fibers have transversely isotropic properties. So, the representative elementary volume method and double homogenization should be used for determining effective elastic constants of three-component composites.

The paper [8] describes an approach to determining effective elastic characteristics of the three-component unidirectional composite with transtropic characteristics of the components reinforced with two types of continuous fibers. The method is based on the application of formulas obtained for a two-phase composite with transtropic properties of the components. This approach has been developed for the case of periodic reinforcement with hollow and continuous transtropic fibers [9].
[10] examines microdeformations of a composite stochastically reinforced with unidirectionally oriented continuous fibers and spheroidal inclusions. In this case, an isotropic matrix and inclusions with various transversely isotropic properties are considered.

The mixture method for determining effective elastic constants of the two-component hollow fiber composite was developed for polyreinforced composites reinforced with $n$ hollow fiber types. The obtained formulas take into account the excellent elastic characteristics of each fiber type. But the use of these relations is limited by the isotropic properties of all the composite components. Considering the transtropic properties of the composite components is not a solved problem.

All this suggests that it is advisable to conduct a study on determining effective characteristics of the three-component composite with transtropic components, unidirectionally reinforced with hollow fibers.

\section{The aim and objectives of the study}

The aim of the study is to determine elastic constants of a unidirectional composite periodically reinforced with two types of transtropic hollow fibers by double homogenization. This will make it possible to take into account a wider range of component properties at the design stage and obtain new composites with predefined properties.

To achieve the aim, the following objectives were set:

- to develop a method for determining the elastic constants of three-component composites based on double homogenization;

- to perform the calculation of elastic constants using the formulas obtained for the two-component composite with the transtropic matrix and hollow fiber.

\section{Research materials and methods}

The object of research is the deformation processes of the three-component composite reinforced with two types of hollow fibers.

The hypotheses include the following: all the composite materials obey Hooke's law. Perfect adhesion between the matrix and fiber materials is assumed.

To develop an approach to determining effective elastic characteristics of the studied composites, the representative elementary volume method is used.

To find effective elastic constants of the three-component composite, the formulas based on the kinematic matching criterion for the two-component unidirectional composite with the transtropic matrix and hollow fiber were used.

Longitudinal Young's modulus [11]:

$$
E_{1}=E_{1}^{*} \frac{\alpha}{d^{\circ} \alpha-\gamma}\left(d^{*} f+d^{\circ}(1-f-g)\right)
$$

where $f$ and $g$ are the volume content of fiber material and hollows in the composite, respectively, $E_{1}^{*}, E_{2}^{*}, v_{12}^{*}, v_{21}^{*}, v_{23}^{*}$ are the elastic characteristics of the matrix material, $E_{1}^{\circ}, E_{2}^{\circ}, v_{12}^{\circ}$, $v_{21}^{\circ}, v_{23}^{\circ}$ are the elastic characteristics of the fiber material, $d^{\circ}$, $d^{*}, \alpha, \beta, \gamma$ are determined by the relations:

$$
d^{\circ}=\frac{\alpha+\beta v_{12}^{\circ}}{\alpha E_{1}^{\circ}},
$$




$$
\begin{aligned}
& d^{*}=\frac{\alpha+\beta v_{12}^{*}}{\alpha E_{1}^{*}}, \\
& \alpha=(1-f-g)\left(\left(E_{2}^{*} v_{23}^{\circ}-E_{2}^{\circ} v_{23}^{*}\right) f-E_{2}^{*}(f+2 g)\right)- \\
& -E_{2}^{\circ}(1+f+g) f, \\
& \beta=2(f+g)\left(v_{21}^{\circ} E_{2}^{*}(1-f-g)+v_{21}^{*} E_{2}^{\circ} f\right), \\
& \gamma=2 f(f+g)\left(v_{21}^{\circ} E_{2}^{*} \frac{v_{12}^{*}}{E_{1}^{*}}-v_{21}^{*} E_{2}^{\circ} \frac{v_{12}^{*}}{E_{1}^{\circ}}\right) .
\end{aligned}
$$

Poisson's ratio [11]:

$v_{12}=\frac{v_{21}^{*} v_{12}^{*} d^{\circ} \alpha-\gamma}{v_{21}^{*}\left(d^{\circ} \alpha-\gamma\right)}$

Longitudinal shear modulus [12]:

$$
G_{12}=\frac{G_{12}^{*}\left(G_{12}^{\circ} f(1+f+g)^{2}+G_{12}^{*}(f+2 g)\left(1-(f+g)^{2}\right)\right)}{G_{12}^{\circ} f\left(1-(f+g)^{2}\right)+G_{12}^{*}(f+2 g)(1+f+g)^{2}} .
$$

Transverse Young's modulus [9]:

- based on the equality of radial displacements

$$
E_{2}=\frac{2 \eta E_{2}^{*}}{\eta\left(4 \gamma_{3}\left(1-v_{21}^{*} v_{12}^{*}\right)+\left(1+v_{23}^{*}\right)+\gamma_{1}\right)+2(f+g)\left(f \gamma_{1} E_{2}^{\circ}-E_{2}^{*}\left((f+2 g) \gamma_{2}+2 g v_{23}^{\circ}\right)\right)}
$$

$$
\begin{aligned}
& \eta=E_{2}^{*}\left(2 g+f\left(1-v_{23}^{\circ}\right)\right)(f+g-1)- \\
& -f E_{2}^{\circ}\left((f+g)\left(1-v_{23}^{*}\right)+\left(1+v_{23}^{*}\right)\right), \\
& \tau=E_{2}^{*}\left(1+v_{23}^{\circ}\right)(f+g-1)+E_{2}^{\circ}\left(\begin{array}{l}
(f+g)\left(1-v_{23}^{*}\right)+ \\
+\left(1+v_{23}^{*}\right)
\end{array}\right),
\end{aligned}
$$$$
\chi_{1}=d_{13} t_{4}-d_{23} t_{3}, \quad \chi_{2}=d_{23} t_{2}-d_{13} t_{1}, \quad \chi_{3}=t_{1} t_{3}-t_{2} t_{4},
$$$$
d_{11}=k_{11}\left(f^{2}+3(f+g)(g-1)\right)-
$$$$
-3 k_{12}(f+g)(g-1)(f+2 g+1) \text {, }
$$

$$
\begin{aligned}
& d_{12}=k_{13}\left((f+g)^{2}+(f+g)(1-3 g)+1\right)- \\
& -3 k_{14}(f+g)(g-1)(f+2 g+1),
\end{aligned}
$$

$$
\begin{aligned}
& d_{13}=k_{13} E_{2}^{*}(f+g-1)\left((f+g)^{2}+f+g+1\right)+ \\
& +E_{2}^{\circ} f\left(\begin{array}{l}
k_{11}\left(f^{2}+3 g(f+g)\right)- \\
-3\left(1+v_{23}^{*}\right)(f+g)^{2}(f+g-1) \times \\
\times(g-1)(f+2 g+1)
\end{array}\right),
\end{aligned}
$$

$$
\begin{aligned}
& d_{21}=k_{21}\left(f^{2}+3(f+g)(g-1)\right)+ \\
& +3 k_{22}(f+g)(g-1)(f+2 g+1),
\end{aligned}
$$

where

$$
\begin{aligned}
& \gamma_{1}=\left(1-v_{23}^{*}\right)-\frac{d_{0} f v_{21}^{*}}{d^{*} f+d^{\circ}(1-f-g)}, \\
& d_{22}=k_{23}\left((f+g)^{2}+(f+g)(1-3 g)+1\right)- \\
& -3 k_{24}(f+g)(g-1)(f+2 g+1) \text {, } \\
& \gamma_{2}=\left(1-v_{23}^{\circ}\right)-\frac{d^{\circ}(f+g-1) v_{21}^{\circ}}{d^{*} f+d^{\circ}(1-f-g)} \\
& \gamma_{3}=\frac{((f+g)(3 g(f+2 g)-2(f+3))-f g) \chi_{1}+f^{2} \chi_{2}+\left((f+g)^{2}+g(f+2 g)\right) \chi_{3}}{3 \chi_{3}(g-1)(f+2 g+1)(f+g-1)}, \\
& \gamma_{4}=\frac{\left((g-1)\left(4(f+g)^{2}+g(f+2 g)\right)-f^{2} g\right) \chi_{1}-f^{2} \chi_{2}-\left((f+g)^{2}+g(f+2 g)\right) \chi_{3}}{3 \chi_{3}(g-1)(f+2 g+1)(f+g-1)}, \\
& d_{23}=k_{23} E_{2}^{*}(f+g-1)\left(\begin{array}{c}
(f+g)^{2}+ \\
+f+g+1
\end{array}\right)+ \\
& +E_{2}^{\circ} f\left(\begin{array}{l}
k_{21}\left(f^{2}+3 g(f+g)\right)- \\
-3\left(1+v_{23}^{*}\right)(f+g)^{2} \times \\
\times(f+g-1) \times \\
\times(g-1)(f+2 g+1)
\end{array}\right), \\
& k_{11}=4(f+g)^{3}\left(v_{23}^{*}+v_{21}^{*} v_{12}^{*}\right)- \\
& d^{\circ}=\frac{v_{21}^{\circ}}{E_{2}^{\circ}}\left(v_{21}^{\circ}\left(\frac{\tau}{\eta} f+1\right)-\frac{2 v_{21}^{\circ}}{1-v_{23}^{\circ}}-2 v_{21}^{*} E_{2}^{\circ} f \frac{f+g}{\eta}\right)- \\
& -\frac{1-v_{23}^{\circ}-2 v_{21}^{\circ} v_{12}^{\circ}}{E_{1}^{\circ}\left(1-v_{23}^{\circ}\right)} \\
& d^{*}=\frac{v_{21}^{*}}{E_{2}^{*}}\left(v_{21}^{\circ}\left(\frac{\tau}{\eta} f+1\right)-\frac{2 v_{21}^{*}}{1-v_{23}^{*}}-2 v_{21}^{*} E_{2}^{\circ} f \frac{f+g}{\eta}\right)- \\
& -\frac{1-v_{23}^{*}-2 v_{21}^{*} v_{12}^{*}}{E_{1}^{*}\left(1-v_{23}^{*}\right)} \text {, } \\
& d_{0}=\left(\frac{v_{21}^{\circ}}{E_{2}^{\circ}} \frac{\tau}{\eta}-2 v_{21}^{*} \frac{f+g}{\eta}\right)\left(\begin{array}{l}
\frac{E_{2}^{\circ}}{E_{2}^{*}} f\left(1-v_{23}^{*}\right)- \\
-f\left(1-v_{23}^{\circ}\right)-2 g
\end{array}\right)- \\
& -\frac{2 v_{21}^{*}}{E_{2}^{*}}+\frac{v_{21}^{\circ}}{E_{2}^{\circ}}\left(1+v_{23}^{\circ}\right)+\frac{v_{21}^{\circ}}{E_{2}^{*}}\left(1-v_{23}^{*}\right) \text {, } \\
& -\left(1+v_{23}^{*}\right)\left(1+3(f+g)^{2}\right) \text {, } \\
& k_{12}=4(f+g)\left(1-v_{21}^{*} v_{12}^{*}\right)+\left(1+v_{23}^{*}\right)\left((f+g)^{2}-1\right), \\
& k_{13}=\left(3 g(f+g)^{2}+g^{3}\right)\left(1+v_{23}^{\circ}\right)- \\
& -4(f+g)^{3}\left(v_{23}^{\circ}+v_{21}^{\circ} v_{12}^{\circ}\right) \text {, } \\
& k_{14}=\left((f+g)^{2}-g^{2}\right)\left(1+v_{23}^{\circ}\right)+4 g(f+g)\left(1-v_{21}^{\circ} v_{12}^{\circ}\right) \text {, } \\
& k_{21}=2(f+g)^{3}\left(3+v_{23}^{*}-2 v_{21}^{*} v_{12}^{*}\right)+ \\
& +\left(1+v_{23}^{*}\right)\left(1-3(f+g)^{2}\right) \text {, } \\
& k_{22}=2(f+g)\left(v_{23}^{*}+2 v_{21}^{*} v_{12}^{*}-1\right)- \\
& -\left(1+v_{23}^{*}\right)\left((f+g)^{2}+1\right) \text {, }
\end{aligned}
$$




$$
\begin{aligned}
& k_{23}=\left(3 g(f+g)^{2}-g^{3}\right)\left(1+v_{23}^{\circ}\right)- \\
& -2(f+g)^{3}\left(3+v_{23}^{\circ}-2 v_{21}^{\circ} v_{12}^{\circ}\right), \\
& k_{24}=\left((f+g)^{2}+g^{2}\right)\left(1+v_{23}^{\circ}\right)- \\
& -2 g(f+g)\left(v_{23}^{\circ}+2 v_{21}^{\circ} v_{12}^{\circ}-1\right), \\
& t_{1}=d_{21} E_{2}^{\circ} f+k_{23} E_{2}^{*}(f+g-1)^{3}, \\
& t_{2}=d_{11} E_{2}^{\circ} f+k_{13} E_{2}^{*}(f+g-1)^{3}, \\
& t_{3}=k_{11} E_{2}^{\circ} f^{3}+d_{12} E_{2}^{*}(f+g-1), \\
& t_{4}=k_{21} E_{2}^{\circ} f^{3}+d_{22} E_{2}^{*}(f+g-1),
\end{aligned}
$$

- based on the equality of circumferential displacements

$$
E_{2}=\frac{2 \eta E_{2}^{*}}{\eta\left(4 \gamma_{4}\left(1-v_{21}^{*} v_{12}^{*}\right)+\left(1+v_{23}^{*}\right)+\gamma_{1}\right)+2(f+g)\left(f \gamma_{1} E_{2}^{\circ}-E_{2}^{*}\left((f+2 g) \gamma_{2}+2 g v_{23}^{\circ}\right)\right)} .
$$

Poisson's ratio [9]:

- based on the equality of radial displacements

$$
v_{23}=\frac{\eta\left(4 \gamma_{3}\left(1-v_{21}^{*} v_{12}^{*}\right)+\left(1+v_{23}^{*}\right)-\gamma_{1}\right)-2(f+g)\left(f \gamma_{1} E_{2}^{\circ}-E_{2}^{*}\left((f+2 g) \gamma_{2}+2 g v_{23}^{\circ}\right)\right)}{\eta\left(4 \gamma_{3}\left(1-v_{21}^{*} v_{12}^{*}\right)+\left(1+v_{23}^{*}\right)+\gamma_{1}\right)+2(f+g)\left(f \gamma_{1} E_{2}^{\circ}-E_{2}^{*}\left((f+2 g) \gamma_{2}+2 g v_{23}^{\circ}\right)\right)}
$$

- based on the equality of circumferential displacements

$$
v_{23}=\frac{\eta\left(4 \gamma_{4}\left(1-v_{21}^{*} v_{12}^{*}\right)+\left(1+v_{23}^{*}\right)-\gamma_{1}\right)-2(f+g)\left(f \gamma_{1} E_{2}^{\circ}-E_{2}^{*}\left((f+2 g) \gamma_{2}+2 g v_{23}^{\circ}\right)\right)}{\eta\left(4 \gamma_{4}\left(1-v_{21}^{*} v_{12}^{*}\right)+\left(1+v_{23}^{*}\right)+\gamma_{1}\right)+2(f+g)\left(f \gamma_{1} E_{2}^{\circ}-E_{2}^{*}\left((f+2 g) \gamma_{2}+2 g v_{23}^{\circ}\right)\right)}
$$

To verify the proposed model, the analytical formulas can be used given in [13]:

$$
\begin{aligned}
& E_{x}=\sum_{i=1}^{n} E_{i} V_{i}\left(1-q_{i}^{2}\right)+E_{m} V_{m}, \\
& \mu_{x y}=\mu_{m}-\frac{\sum_{i=1}^{n} \frac{V_{i}\left(1-q_{i}^{2}\right)\left(\mu_{m}-\mu_{i}\right)\left(\chi_{m}+1\right)}{2\left(1-q_{i}^{2}\right)+\left(\chi_{i}-1+2 q_{i}^{2}\right) G_{m} / G_{i}}}{V_{m}+\sum_{i=1}^{n} \frac{V_{i}\left(1-q_{i}^{2}\right)\left(\chi_{m}+1\right)}{2\left(1-q_{i}^{2}\right)+\left(\chi_{i}-1+2 q_{i}^{2}\right) G_{m} / G_{i}}} \\
& E_{y}=\left(\frac{\mu_{x y}^{2}}{E_{x}}+\frac{\chi_{m}+1}{8 G_{m}}\left(\frac{1}{N}-\frac{2 Q}{1+Q}\right)\right)^{-1}, \\
& \frac{\mu_{z y}}{E_{y}}=-\frac{\mu_{x y}^{2}}{E_{x}}+\frac{\mu_{m}}{2 G_{m}}+\frac{\chi_{m}+1}{8 G_{m}}\left(\frac{N-1}{N}-\frac{2 Q}{1+Q}\right) \\
& G_{x y}=G_{m} \frac{\left(1-q_{i}^{2}\right) V_{i}}{1-\sum_{i=1}^{n} V_{i} \frac{1-q_{i}^{2}-\left(1+q_{i}^{2}\right) G_{m} / G_{i}}{1-q_{i}^{2}+\left(1+q_{i}^{2}\right) G_{m} / G_{i}}}
\end{aligned}
$$

where $V_{m}, \chi_{i}, \chi_{m}, Q, N$ are determined by the relations:

$$
V_{m}=1-\sum_{i=1}^{n} V_{i}
$$

\section{Mathematical modeling of effective elastic characteristics of the three-component composite}

5. 1. Method of numerical calculation of effective elastic constants of the three-component composite

The unidirectional composite reinforced with two types of hollow fibers by the hexagonal scheme is considered. The study is conducted within the linear theory of elasticity. Perfect adhesion at the matrix-hollow fiber interface is assumed.

The cross-section of the composite is shown in Fig. 1, $a$. Here, the whole area is divided into hexagonal cells so that the center of each hexagon coincides with the fiber center. Two types of hexagonal cells are obtained: for type I hollow fiber with the surrounding matrix (indicated by diagonal hatching) and type II hollow fiber with the surrounding matrix (indicated by crosshatching). Type I isotropic fiber is characterized by the following elastic characteristics: elastic modulus $E_{(1)}^{\circ}$, Poisson's ratio $v_{(1)}^{\circ}$. In the case of transtropic components, we have elastic constants: longitudinal Young's modulus $E_{1(1)}^{\circ}$, Poisson's ratio $v_{12(1)}^{\circ}$, transverse Young's modulus $E_{2(1)}^{\circ}$, Poisson's ratio $v_{23(1)}^{\circ}$, longitudinal shear modulus $G_{12(1)}^{\circ}$. Type II isotropic fiber is characterized similarly by: elastic modulus $E_{(2)}^{\circ}$, Poisson's ratio $v_{(2)}^{\circ}$ (in the case of transtropic components, $E_{1(2)}^{\circ}$, $\left.v_{12(2)}^{\circ}, E_{2(2)}^{\circ}, v_{23(2)}^{\circ}, G_{12(2)}^{\circ}\right)$. The isotropic matrix surrounding each of the two fiber types is characterized by elastic modulus $E^{*}$ and Poisson's ratio (in the case of transtropic components $\left.E_{1}^{*}, v_{12}^{*}, E_{2}^{*}, v_{23}^{*}, G_{12}^{*}\right)$.

In the first step, the hexagonal regions with type I and II fibers are separately homogenized. The hexagonal cell is approximated by a circle so that its area and the cell area are the same.

First, according to formulas (1), (3)-(8), effective elastic constants of the first region are calculated, substituting elastic constants of the matrix and type I fiber in them. Then, according to the same formulas, effective elastic constants of the second region, substituting elastic constants of the matrix and type II fiber in them.

Thus, a "conditional" two-component composite with a "conditional" fiber (type I fiber regions before the first homogenization) and a "conditional" matrix (type II fiber regions, respectively) are obtained (Fig. 1, $b$ ). 


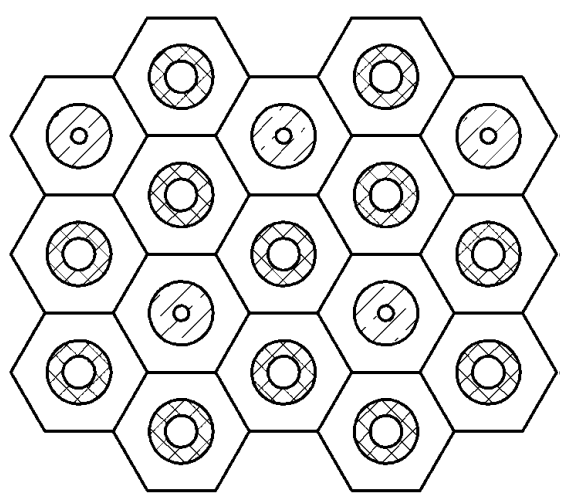

$a$

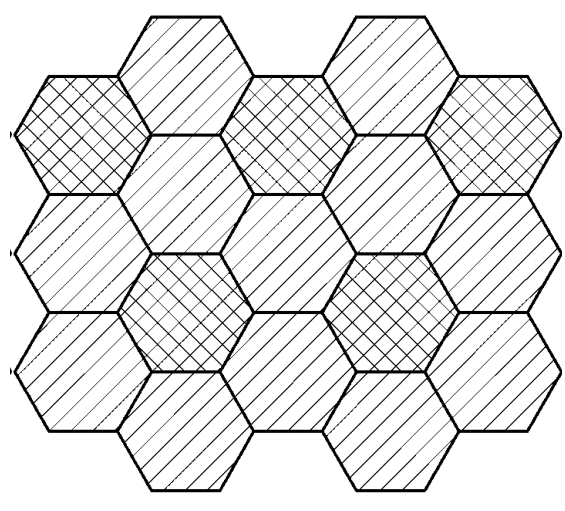

$b$

Fig. 1. Representation of the cross-section of the three-component composite: $a$ - before the "first" homogenization; $b$ - after the "first" homogenization

This technique is valid for high fiber reinforcement frequency, when the number of unit cells is tens or more both in length and width. With such periodic reinforcement, the ratio of the type I cell area $S_{(1)}$ ("conditional" fiber) to the type II cell area $S_{(2)}$ ("conditional" matrix) tends to 3.

Then, the total cross-sectional area of the composite after the first homogenization $S$ is:

$$
S=n\left(S_{(1)}+3 S_{(2)}\right)=n\left(S_{(1)}^{*}+S_{(1)}^{\circ}+3 S_{(2)}^{*}+3 S_{(2)}^{\circ}\right),
$$

where $4 n$ is the total number of cells, $S_{(1)}^{*}$ and $S_{(1)}^{\circ}$ are the areas of the matrix material and the first-type fiber material in the "conditional" fiber, $S_{(2)}^{*}, S_{(2)}^{\circ}$ are the areas of the matrix material and the second-type fiber material in the "conditional" matrix.

Using the representation of the hexagonal cell as a circle containing a hollow fiber, where $a_{(i)}$ is the radius of the $i$-th type hollow fiber, $b$ is the circle radius, and from (15), we obtain:

$$
S=n \pi\left(\left(b^{2}-a_{(1)}^{2}\right)+a_{(1)}^{2}+3\left(b^{2}-a_{(2)}^{2}\right)+3 a_{(2)}^{2}\right) .
$$

On the other hand, the total area is $S=4 n \pi b^{2}$. Then

$$
1=t^{*}+t_{(1)}^{\circ}+t_{(2)}^{\circ}
$$

where $t^{*}, t_{(1)}^{\circ}, t_{(2)}^{\circ}$ are the volume fractions of the matrix, first and second-type hollow fiber, respectively, in the initial three-component material and are determined by the relations:

$$
\begin{aligned}
& t^{*}=1-\frac{a_{(1)}^{2}+3 a_{(2)}^{2}}{4 b^{2}}, \\
& t_{(1)}^{\circ}=\frac{a_{(1)}^{2}}{4 b^{2}}, \\
& t_{(2)}^{\circ}=\frac{3 a_{(2)}^{2}}{4 b^{2}} .
\end{aligned}
$$

The volume fraction of the first-type hollow fiber in the "conditional" fiber is then defined as

$$
q_{(1)}^{\circ}=4 t_{(1)}^{\circ} \text {, }
$$

and the volume fraction of the second-type hollow fiber in the "conditional" matrix

$$
q_{(2)}^{\circ}=\frac{4}{3} t_{(2)}^{\circ}
$$

At the same time, $t_{(1)}^{\circ}=f_{1}+g_{1}$ for the first-type fiber, and $t_{(2)}^{\circ}=f_{2}+g_{2}$ for the second-type fiber. Here, $f_{1}, f_{2}$ are the volume fractions of the first and second-type fiber material, respectively, $g_{1}, g_{2}$ are the volume fractions of the hollow in the fiber of each type.

At the second stage, repeated homogenization is performed. The effective elastic constants of the three-component composite $E_{1}, v_{12}, G_{12}, E_{2}, v_{23}$ are determined by the same formulas. The input data for the "conditional" fiber are the elastic characteristics $E_{1}^{(\circ)}, v_{12}^{(\circ)}, G_{12}^{(\circ)}, E_{2}^{(\circ)}, v_{23}^{(\circ)}$, and for the "conditional" matrix $E_{1}^{(*)}, v_{12}^{(*)}, G_{12}^{(*)}, E_{2}^{(*)}, v_{23}^{(*)}$ obtained at the first stage.

Verification of the presented method is implemented by calculating effective elastic constants of fiberglass based on EDT-10 epoxy binder with the elastic properties: longitudinal Young's modulus $E^{*}=2.9$ GPA, Poisson's ratio $v^{*}=0.35$ [14]. As reinforcing hollow fibers, aluminum-borosilicate glass fibers with a paraffin-emulsion lubricant and VM-1 high-modulus glass fibers with a 752 lubricant are taken, the elastic properties of which are, respectively: $E_{(1)}^{\circ}=73.1 \mathrm{GPA}, v_{(1)}^{\circ}=0.25, E_{(2)}^{\circ}=100 \mathrm{GPA}, v_{(2)}^{\circ}=0.25$.

At the first stage, the elastic constants of the "conditional" fiber and "conditional" matrix are calculated. For calculations, we fix $t_{(1)}^{\circ}=0.05\left(g_{1}=0.02\right)$, and take $t_{(2)}^{\circ}$ in the range of $0.1 ;. . ; 0.3$ with a step of $0.1\left(g_{2}=0.05\right)$.

At the second stage, the elastic characteristics of the composite are calculated from the obtained data (transtropic characteristics of the "conditional" matrix and "conditional" fiber) at $g=0$ and $f=0.25$.

Compare the values of longitudinal Young's modulus $E_{1}$, Poisson's ratio $v_{12}$, and transverse Young's modulus $E_{2}$ calculated by the developed method and formulas (9)-(11). The calculation results are summarized in Table 1.

Table 1

Values of longitudinal Young's modulus $E_{1}$, Poisson's ratio $v_{12}$, and transverse Young's modulus $E_{2}$ calculated by formulas (9)-(11) and (1), (3), (5), (6)

\begin{tabular}{|l|c|c|c|c|c|c|c|c|}
\hline \multicolumn{2}{|c|}{$\begin{array}{c}\text { Volume } \\
\text { fraction }\end{array}$} & \multicolumn{2}{|c|}{$E_{1}, \mathrm{GPa}$} & \multicolumn{2}{|c|}{$v_{12}$} & \multicolumn{3}{|c|}{$E_{2}, \mathrm{GPa}$} \\
\hline$t_{(1)}^{\circ}$ & $t_{(2)}^{\circ}$ & $(9)$ & $(1)$ & $(10)$ & $(3)$ & $(11)$ & $(5)$ & $(6)$ \\
\hline 0.05 & 0.1 & 9.658 & 9.663 & 0.33213 & 0.33212 & 3.849 & 3.356 & 3.329 \\
\hline 0.05 & 0.2 & 19.368 & 19.375 & 0.32044 & 0.32043 & 4.653 & 5.031 & 5.448 \\
\hline 0.05 & 0.3 & 29.078 & 29.087 & 0.30945 & 0.30937 & 5.591 & 6.067 & 7.276 \\
\hline
\end{tabular}


The data shown in Table 1 show a good agreement between the calculation results of the above method and the mixture method [13] for a composite with an isotropic matrix and two types of hollow fibers. Thus, the maximum relative calculation error of the proposed method for $E_{1}$ compared to formula (9) does not exceed $0.05 \%$. The maximum relative calculation error for the Poisson's ratio $v_{12}$ compared to formula (10) does not exceed $0.03 \%$.

The above method pays special attention to determining the transverse characteristics, namely transverse Young's modulus $E_{2}$ and Poisson's ratio $v_{23}$. Accordingly, the greatest errors are obtained when calculating these characteristics. This method has been tested for other fiber types with experimental data [8], and shows greater convergence of results than calculations by [13] and the proposed approach.

\section{2. Numerical determination of effective elastic con-} stants of the three-component composite

For the three-component composite from [14], Fig. 2-6 graphically present the dependences of some effective elastic constants of the three-component composite on the volume content of the second-type hollow fiber $t_{(2)}^{\circ}$ with a fixed volume fraction of the first-type hollow fiber $t_{(1)}^{\circ}=0.05$.

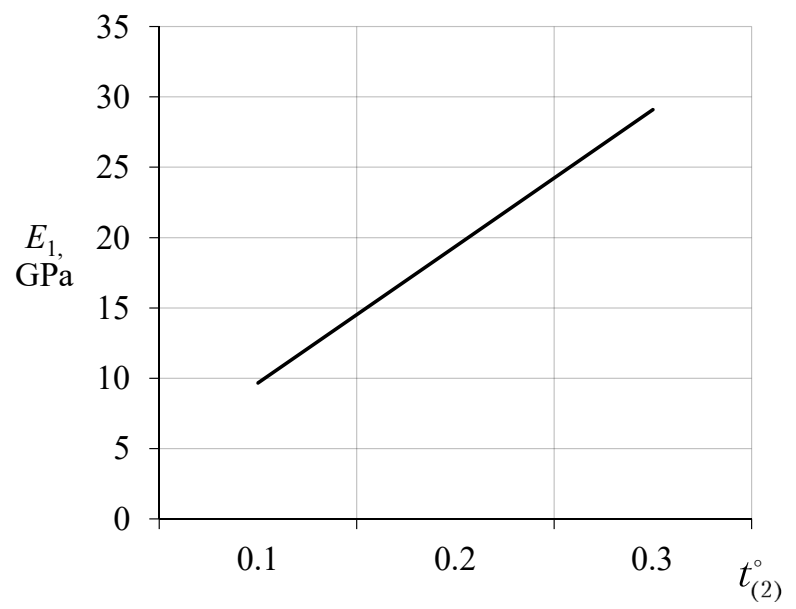

Fig. 2. Dependence of longitudinal Young's modulus $E_{1}$ on the volume fraction of the second-type hollow fiber $t_{(2)}^{\circ}$

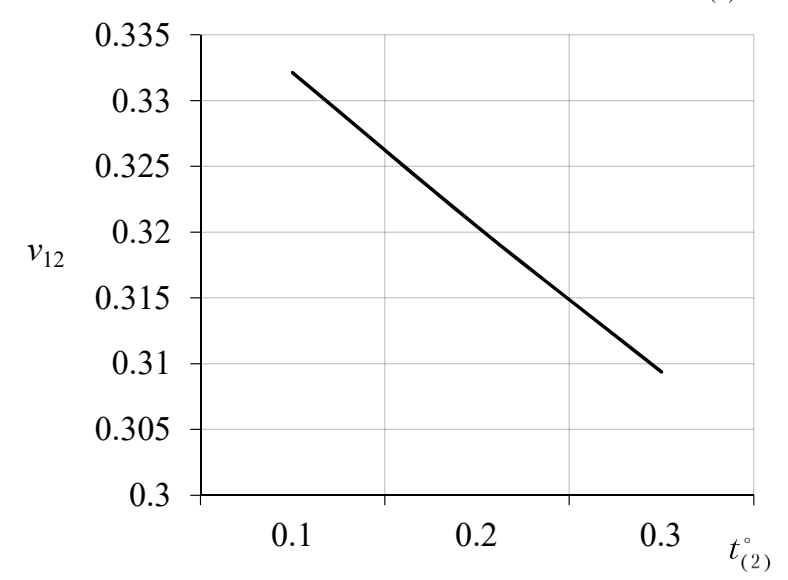

Fig. 3. Dependence of Poisson's ratio $v_{12}$ on the volume fraction of the second-type hollow fiber $t_{(2)}^{\circ}$

The above graphs (Fig. 2,3) suggest that the longitudinal Young's modulus $E_{1}$ and the Poisson's ratio $v_{12}$ have a close to linear dependence on the volume content of the second-type hollow fiber. An increase in the volume fraction $t_{(2)}^{\circ}$ increases the transverse Young's modulus $E_{2}$ (Fig. 4), longitudinal shear modulus $G_{12}$ (Fig. 5) and Poisson ratio $v_{23}$ (Fig. 6).

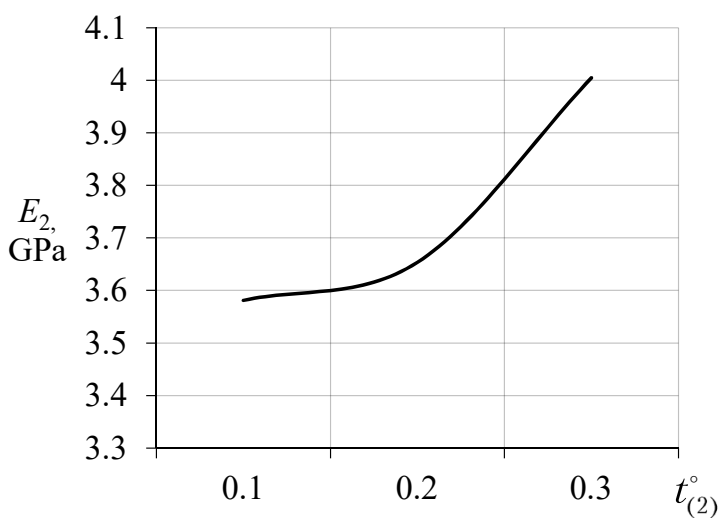

Fig. 4. Dependence of transverse Young's modulus $E_{2}$ on the volume fraction of the second-type hollow fiber $t_{(2)}^{\circ}$

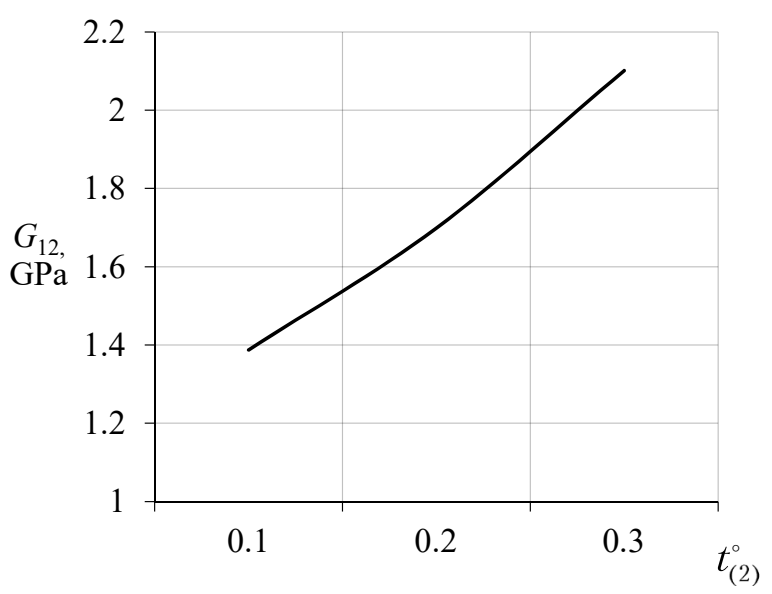

Fig. 5. Dependence of longitudinal shear modulus $G_{12}$ on the volume fraction of the second-type hollow fiber $t_{(2)}^{\circ}$

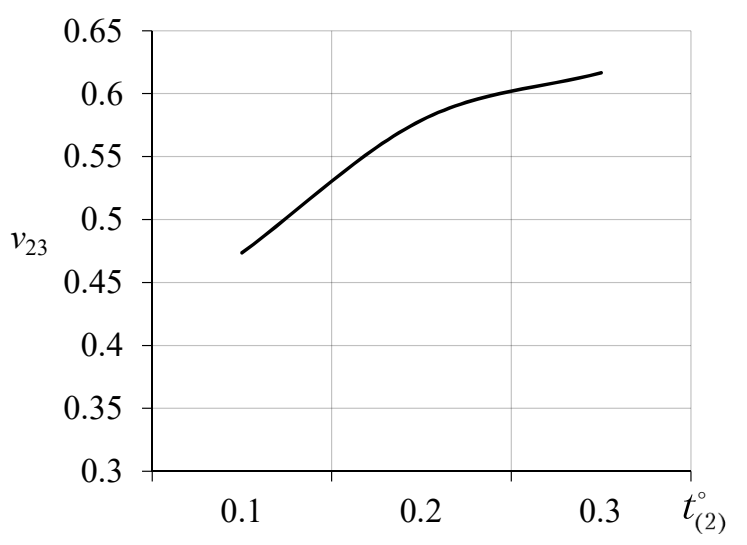

Fig. 6. Dependence of the Poisson's ratio $v_{23}$ on the volume fraction of the second-type hollow fiber $t_{(2)}^{\circ}$

\section{Discussion of the results of applying the developed method}

The values of effective elastic constants obtained by the proposed method (Table 1) agree well with the calculations by formulas [13]. In particular, the maximum relative calculation error for the longitudinal elastic characteristics does not exceed $0.05 \%$. In the partial case (if the same fiber characteristics are taken and elastic constants are calculated by 
the proposed approach for the three-component composite), adequate results are also obtained.

The proposed method applies only to unidirectional three-component composites with hexagonal lay-up of two types of hollow fibers for deformations within Hooke's law. A feature is the periodic reinforcement structure, i.e. when a cell with one fiber type is surrounded by six cells with fibers of another type.

The analysis of the graphical research results (Fig. 2-6) reveals the effect of hollow size on the effective elastic constants of the studied composites.

The advantage of the developed method is the possibility of using it for composites reinforced with two types of hollow fibers, both with isotropic and transtropic properties of the components. This was not taken into account earlier in [13].

It should be noted that the method is based on the formulas derived from the kinematic matching criterion. Therefore, to determine the transverse Young's modulus $E_{2}$ and Poisson's ratio $v_{23}$, a certain pair of formulas should be chosen within the proposed approach. This choice depends on experimental data for each individual composite, depending on the elastic properties of its components.

It is promising to obtain relations based on the energy criterion and improve the developed method based on five rather than seven formulas for determining the effective elastic constants. This approach gives the best result com- pared to experimental data. However, the application of the representative elementary volume method with this approach is significantly complicated by the bulkiness of the mathematical model. The above approach can be generalized for arbitrary load types and fiber lay-up patterns.

\section{Conclusions}

1. The method for determining the elastic constants of three-component composites unidirectionally reinforced with two types of hollow fibers with transtropic characteristics of the components based on double homogenization is developed. The method is based on the formulas obtained for a unidirectional two-phase composite with the transtropic matrix and hollow fiber. The maximum relative calculation error for the longitudinal Young's modulus and Poisson's ratio compared to known formulas does not exceed $0.05 \%$.

2. The values of effective elastic constants are calculated by the proposed method using the example of a three-component composite with isotropic properties of the components. The dependences of elastic characteristics on the volume content of the second-type hollow fiber are constructed. In particular, the transverse Young's modulus $E_{2}$ increases by about $10 \%$ with an increase in the hollow fiber volume fraction from 0.1 to 0.3 , while the longitudinal Young's modulus $E_{1}-$ about three times.

\section{References}

1. Wang, G., Tu, W., Pindera, M.-J. (2017). Tailoring the moduli of composites using hollow reinforcement. Composite Structures, 160 , 838-853. doi: https://doi.org/10.1016/j.compstruct.2016.10.060

2. Balaji, R., Sasikumar, M., Jeyanthi, S. (2016). Characterisation of hollow glass fibre reinforced vinyl-ester composites. Indian Journal of Science and Technology, 9 (47), 1-5. doi: https://doi.org/10.17485/ijst/2016/v9i48/107921

3. Nasr-Isfahani, M., Tehran, M. A., Latifi, M., Halvaei, M., Warnet, L. (2017). Experimental and theoretical investigation of hollow polyester fibers effect on impact behavior of composites. Journal of Industrial Textiles, 47 (7), 1528-1542. doi: https://doi.org/ $10.1177 / 1528083717699367$

4. Bayat, M., Aghdam, M. M. (2012). A micromechanics based analysis of hollow fiber composites using DQEM. Composites Part B: Engineering, 43 (8), 2921-2929. doi: https://doi.org/10.1016/j.compositesb.2012.06.021

5. Nasr-Isfahani, M., Latifi, M., Amani-Tehran, M. (2013). Improvement of Impact Damage Resistance of Epoxy-Matrix Composites Using Ductile Hollow Fibers. Journal of Engineered Fibers and Fabrics, 8 (1), 155892501300800. doi: https://doi.org/ $10.1177 / 155892501300800108$

6. Naeimirad, M., Abuzade, R., Babaahmadi, V., Neisiany, R. E., Brüll, R., Pursche, F. (2021). Hollow fiber reinforced polymer composites. Fiber Reinforced Composites, 461-477. doi: https://doi.org/10.1016/b978-0-12-821090-1.00001-6

7. Aimmanee, S., Asanuma, H. (2019). Micromechanics-based predictions of effective properties of a 1-3 piezocomposite reinforced with hollow piezoelectric fibers. Mechanics of Advanced Materials and Structures, 27 (22), 1873-1887. doi: https://doi.org/10.1080/ 15376494.2018.1529842

8. Grebeniuk, S. (2016). Effective elastic constants of the composite material reinforced by the unidirectional fibers of the two types. Visnyk of Zaporizhzhya National University. Physical and Mathematical Sciences, 1, 48-56. Available at: http://journalsofznu.zp.ua/ index.php/phys-math/article/view/1342/1295

9. Stoliarova, A. V., Koval, R. A., Hatsenko, A. V., Dioba, N. O. (2021). The determination of the elastic constants of the composite material with solid and hollow equivalently directed fibers. Bulletin of Zaporizhzhia National University. Physical and Mathematical Sciences, 1, 57-64. doi: https://doi.org/10.26661/2413-6549-2021-1-07

10. Nazarenko, L. V. (2008). Deformative properties of granular-fiber composites under matrix microdamaging. Prykladni problemy mekhaniky i matematyky, 6, 146-153. Available at: http://dspace.nbuv.gov.ua/handle/123456789/7704

11. Homeniuk, S., Grebenyuk, S., Klimenko, M., Stoliarova, A. (2018). Determining the effective characteristics of a composite with hollow fiber at longitudinal elongation. Eastern-European Journal of Enterprise Technologies, 6 (7 (96)), 6-12. doi: https://doi.org/ 10.15587/1729-4061.2018.143406

12. Grebenyuk, S., Klymenko, M., Stoliarova, A., Titova, O. (2019). Longitudinal shear modulus of the composite material with hollow fibers. Mechanika 2019: Proceedings of the 24th International Scientific Conference. Kaunas, 45-48. Available at: https://www. researchgate.net/publication/354890490_Longitudinal_Shear_Modulus_of_the_Composite_Material_with_Hollow_Fibers

13. Karpinos, D. M. (Ed.) (1985). Kompozicionnye materialy. Kyiv: Naukova dumka, 588.

14. Tarnopol'skiy, Yu. M., Zhigun, I. G., Polyakov, V. A. (1987). Prostranstvenno-armirovannye kompozicionnye materialy. Moscow: Mashinostroenie, 224. 